\title{
The Clock-Aided Method for GPS Receiver Positioning in an Urban Environment
}

\author{
Yunlong Teng, Yibing Shi, and Zhi Zheng
}

\begin{abstract}
As the signals of GPS satellites are susceptible to obstructions in an urban environment with many tall buildings around, the number of visible satellites is usually less than four. In the circumstances, the normal positioning method can not be used for positioning calculation. In order to obtain continuous positioning results under three satellites, the series of receiver clock bias (RCB) is considered as one visible satellite in this paper. A combined model for predicting the RCB series based on the theory of time series analysis is presented firstly. And then the model is used to aid the normal positioning method for positioning calculation under the conditions of three satellites. From both static and dynamic experiments, it is shown that the prediction model is fit for predicting the RCB series, and the clock-aided positioning method aiding of the prediction model can implement the function of three-dimensional positioning calculation even if there are only three visible satellites.
\end{abstract}

Index Terms-Urban environment, Positioning calculation, Receiver clock bias (RCB), Combined prediction model

\section{INTRODUCTION}

As a high-precision positioning technology, the Global Positioning System (GPS) plays an important role in our daily lives [1]. Normally, there must be at least four satellites in view so that the positioning results of the GPS receivers can be obtained [2,3]. However, in certain applications, for example in an urban environment with many tall buildings around, there are situations wherein the requirement of a minimum of four satellites is not satisfied because of the obstructions to receiver-to-satellite line of sights. In this case, the traditional method cannot be used for calculating the position of GPS receiver anymore. How to deal with this bad-conditioned problem so that the GPS receiver can provide continuous and reliable positioning information is the main theme of this paper.

In order to obtain the continuous and reliable positioning information of GPS receivers under bad conditions, several methods have been presented. Jan solved the problem from three satellites and altimeter [4], Hong combined GPS

Manuscript received January 15, 2011; revised April 18, 2011.This work was supported in part by the Fundamental Research Funds for the Central Universities (ZYGX2009J092) and the Aeronautic Science Foundation of China (20090580013).

Yunlong Teng is with the School of Automation Engineering, and Research Institute of Electronic Science and Technology, University of Electronic Science and Technology of China, Chengdu 611731, Sichuan, China (corresponding author, e-mail: tyluestc@163.com).

Yibing Shi is with the School of Automation Engineering, University of Electronic Science and Technology of China, Chengdu 61173, Sichuan, China (e-mail: ybshi@uestc.edu.cn).

Zhi Zheng is with the Research Institute of Electronic Science and Technology, University of Electronic Science and Technology of China, Chengdu 611731, Sichuan, China (e-mail: zhengzhi-uestc@sohu.com). receiver with the Inertial navigation system [5]. Liu made use of the additional information provided by pseudolite [6]. If a road map database exists, the map-matching technique can also be utilized [7]. The common characters of these methods need external hardware. The above mentioned methods may be useful, but not efficient. In fact, the cost of them has great impact on their popularization.

Actually, besides the position information, the clock bias between GPS receiver and GPS system is given when the function of positioning calculation is executed normally. The bias is called receiver clock bias and short for RCB in the paper. If the clock frequency keeps steady, a prediction model of the RCB series can be obtained by using a length of history data [8]. Hence, the RCB series can be regarded as one visible satellite, and the prediction model on it is then introduced into GPS receiver for positioning calculation under the conditions of only three satellites. Compared with other auxiliary methods, the clock-aided positioning method needs no external equipments. The advantages of the clock-aided positioning method are clear and obvious.

There are many complicated factors which have an impact on the RCB series, including the atmosphere and ionosphere delays, the orbital errors of GPS satellites, etc. Under the influence of these factors, the RCB series can be considered to be nonlinear time series. Generally, prediction models for nonlinear time series can be grouped into two types, single models and combined models. Combined models integrate several single models to improve the prediction precision. Most combined models are decomposition based. They decompose the original time series into several isolated components firstly. And then they employ a single model to predict each of them, and lastly they integrate the results to gain the final prediction value. Because of the approximate of one-fold property of each component, their prediction will be improved [9]. A combined prediction model can capture many more of these properties than a single model, and thus might improve the prediction precision.

Considered the nonlinear characters of the RCB series, a combined model for predicting it based on the theory of time series analysis is presented in the paper. The basic ideas and detailed steps of the combined prediction model are discussed respectively. Then the prediction model is introduced into GPS receiver for positioning calculation together with three visible satellites. Test results are investigated to demonstrate the feasibility and validity of the method in the end.

\section{Design SCHEME OF RCB Prediction Model}

The combined prediction model for the RCB series on the basis of the theory of time series analysis includes three 
phases. Firstly, the RCB series were decomposed into a low- frequency component (LFC) and several high-frequency components (HFCs) by wavelet transform. Then the LFC was predicted through polynomial model (PM) and the HFCs were predicted by using auto-regressive (AR) models. The predicted results were the superimposition of the respective prediction. Based on these procedures, Fig.1 illustrates the flowchart of the combined prediction model.

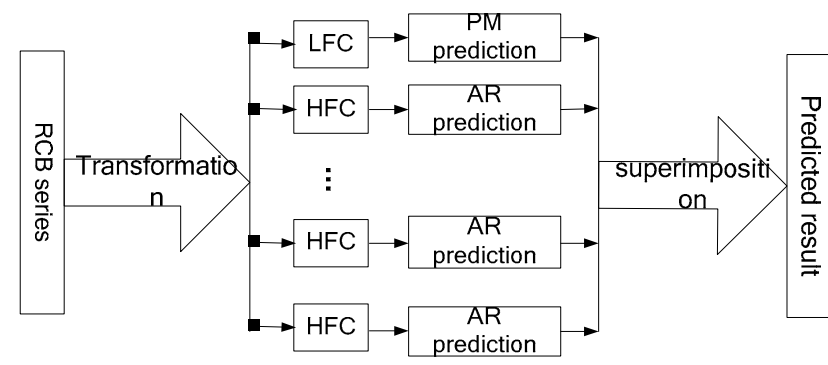

Fig.1 Flowchart of the combined prediction model.

\section{A. Wavelet Transform of RCB Series}

In the combined prediction model, the decomposition method used is wavelet transform for its simplicity. There are continuous wavelets transforms and discrete wavelet transforms. Since the RCB series are discrete, the discrete wavelet transform is adopted. The Mallat algorithm [10] based on multi-resolution representation is a fast discrete wavelet transform algorithm, and it has shown promising results in various areas $[11,12]$. On the basis of the characters of the Mallat algorithm, it is utilized to decompose the RCB series.

Let $\{C(t)\}(t=1 \sim N)$ denote the original RCB series, the decomposition procedure can be described as follows.

$$
\left\{\begin{array}{l}
c_{0}=C \\
c_{k+1}=H c_{k}, \quad k=0,1, \cdots,(K-1) \\
d_{k+1}=G c_{k}
\end{array}\right.
$$

In (1), $\mathrm{K}=\log _{2} \mathrm{~N}$ denotes the maximal decomposition level. $H$ and $G$ are the wavelet filters for decomposition in the time domain, that is to say, $H$ is the low-pass filter and $G$ is the high-pass filter. $c_{k}$ and $d_{k}$ are the LFC and HFC at the corresponding level, respectively. Through iteration, the RCB series could be decomposed into one LFC plus a set of HFCs [11]. The LFC represents the trend term, and the HFC is the stationary component with the zero mean.

After the wavelet decomposition, the lengths of the LFC and HFCs are halved compared with the original series. So they have to be reconstructed by using (2).

$$
D_{k+1}=H^{*} C_{k}+G^{*} D_{k}, \quad k=(K-1), \cdots, 0
$$

Where $H^{*}$ and $G^{*}$ are the dual operators of $H$ and $G$. By means of using (2), $c_{k}\left(d_{k}\right)$ can be reconstructed as $C_{k}\left(D_{k}\right)$, whose lengths equal to the one of the RCB series. And they satisfy $C(t)=C_{K}(t)+D_{1}(t)+\cdots+D_{K}(t)$.

\section{B. The Prediction of $L F C$}

The LFC reveals the development trend of the RCB series with respect to time. Related experiments have demonstrated that when the clock frequency of GPS receiver keeps stable, the RCB series can be modeled and fitted by a polynomial model [8]. Accordingly, the paper takes advantage of the polynomial model to distill the trend of the RCB series, and obtain the predicted series $\bar{C}_{K}(t)$.

\section{The Prediction of HFC}

As discussed above, the HFCs $\left(D_{1}(t), \cdots D_{K}(t)\right)$ obtained from the wavelet transform have the statistics characters, and can be regarded as stationary series. The AR model is the basic and important time series model, which is mostly used for modeling and predicting stationary time series. Hence, AR model is established for predicting the above HFCs. In this section, we take example for $D_{1}(t)$, and the modeling procedures of other HFCs $\left(D_{2}(t), \cdots D_{K}(t)\right)$ are similar. For convenient for further research, $D_{1}(t)$ is short for $x(t)$ in this section. The mathematical expression of AR model for predicting $x(t)$ can be described as

$$
x(t)=\sum_{k=1}^{p} \phi_{k} x(t-k)+\mu(t)
$$

Where $p$ is the order of this model, and it can be determined by employing the AIC method. The parameters $\phi_{k}$ can be calculated by using the Least-square method in (4).

$$
\left[\begin{array}{lll}
\bar{\phi}_{1} & \cdots & \bar{\phi}_{p}
\end{array}\right]=\left(V^{\mathrm{T}} V\right)^{-1} V R
$$

Where

$$
V=\left[\begin{array}{ccc}
x(p) & \cdots & x(1) \\
x(p+1) & \cdots & x(2) \\
\vdots & & \vdots \\
x(N-1) & \cdots & x(N-p)
\end{array}\right], \quad R=\left[\begin{array}{c}
x(p+1) \\
x(p+2) \\
\vdots \\
x(N)
\end{array}\right]
$$

On the basis of the above expressions, we can get the predicted results of $x(t)$.

$$
\bar{x}(t)=\sum_{k=1}^{p} \bar{\phi}_{p} \bar{x}(t-k)
$$

\section{Prediction of RCB Series}

This step is dedicated to calculate the predicted results of the RCB series. By means of combining the predicted results of all the components, we can obtain the underlying predicted results of the RCB series.

$$
\bar{C}(t)=\bar{C}_{K}(t)+\sum_{k=1}^{K} \bar{D}_{\mathrm{k}}(t)
$$

\section{GPS POSITIONING USING CLOCK-ADIED METHOD}

The GPS positioning method aiding of the RCB prediction model is given in this section. We begin with showing the normal positioning method of GPS receiver. Then the clockaided positioning method under the conditions of three visible satellites is discussed particularly.

\section{A. Normal Positioning Method}

Suppose $p_{i}$ denotes the pseudorange from GPS receiver at $(x, y, z)$ to GPS satellite at $\left(x_{i}, y_{i}, z_{i}\right)$. After the modeled errors are properly compensated, the pseudorange between 
them can be written as

$$
p_{i}=\left[\left(x_{i}-x\right)^{2}+\left(y_{i}-y\right)^{2}+\left(z_{i}-z\right)^{2}\right]^{1 / 2}+c+\omega_{i}
$$

Where $c$ denotes the RCB value expressed in units of length, and $\omega_{i}$ is the measurement error. In (8), $x, y, z$ and $c$ are four parameters need to be estimated.

During the course of positioning calculation, (8) should be linearized through Taylor's series around the approximate coordinate $(\bar{x}, \bar{y}, \bar{z})$ and neglecting the higher order terms. Defining $\bar{p}_{i}$ as $p_{i}$ at the approximate coordinate, then we can obtain

$$
\Delta p_{i}=p_{i}-\bar{p}_{i}=a_{x i} \Delta x+a_{y i} \Delta y+a_{z i} \Delta z
$$

Where

$$
\left\{\begin{array}{l}
\Delta \varsigma=\varsigma-\bar{\varsigma}, \quad \varsigma=x, y, z \\
a_{\varsigma i}=\frac{\varsigma_{i}-\bar{\varsigma}}{\bar{p}_{i}}, \quad \varsigma=x, y, z \\
\bar{p}_{i}=\left[\left(x_{i}-\bar{x}\right)^{2}+\left(y_{i}-\bar{y}\right)^{2}+\left(z_{i}-\bar{z}\right)^{2}\right]^{1 / 2}
\end{array}\right.
$$

Let $M$ represent to the number of visible satellites, then we can obtain the linear measurement equation in a compact matrix formulation as

$$
Y=A X+V
$$

In (11), the measurement vector $Y$, the measurement matrix $A$, and the four-dimensional estimated vector $X$ are defined by

$$
Y=\left[\begin{array}{c}
\Delta p_{1} \\
\Delta p_{2} \\
\cdots \\
\Delta p_{M}
\end{array}\right], \quad A=\left[\begin{array}{cccc}
a_{x 1} & a_{y 1} & a_{z 1} & -1 \\
a_{x 2} & a_{y 2} & a_{z 2} & -1 \\
\ldots & \ldots & \ldots & \ldots \\
a_{x M} & a_{y M} & a_{z M} & -1
\end{array}\right], \quad X=\left[\begin{array}{c}
\Delta x \\
\Delta y \\
\Delta z \\
c
\end{array}\right]
$$

Vector $X$ includes four parameters. It is clear that fewer than four satellites will not provide enough information for an acceptable position of GPS receiver. Unfortunately, for car navigation in an urban environment with many tall buildings around, it frequently occurs that there are only three visible satellites. In the circumstances, the normal positioning method cannot solve the four parameters.

\section{B. GPS Positioning Using Clock-Aided Method}

In order to solve the problem of GPS positioning under such bad conditions, the RCB prediction model is considered to be one visible satellite and used to complete the positioning process by expanding the linear measurement equation.

When the clock-aided positioning method is adopted to calculate the position of GPS receiver, the predicted value of the RCB series $\bar{C}(N+1)$ is used to replace the actual one. When there are three visible satellites, the expended equation for positioning calculation can be expressed by the following equation

$$
\bar{Y}=\bar{A} \bar{X}+\bar{V}
$$

In (13), vector $\bar{X}$ only includes three modified items to the approximate coordinate $(\Delta x, \Delta y, \Delta z)$. The expanded measurement vector $\bar{Y}$ and the expanded measurement matrix $\bar{A}$ are described in (14).

$$
\bar{Y}=\left[\begin{array}{c}
\Delta p_{1}-\bar{C}(N+1) \\
\Delta p_{2}-\bar{C}(N+1) \\
\Delta p_{3}-\bar{C}(N+1)
\end{array}\right], \quad \bar{A}=\left[\begin{array}{ccc}
a_{x 1} & a_{y 1} & a_{z 1} \\
a_{x 2} & a_{y 2} & a_{z 2} \\
a_{x 3} & a_{y 3} & a_{z 3}
\end{array}\right]
$$

From (11) and (13), it can be seen that the estimated parameters decrease from four to three after introducing the RCB prediction model. Accordingly, the clock-aided method can obtain the position of GPS receiver even if there are only three visible satellites.

When the inverse of $\bar{A}$ exists, the solution of $\bar{X}$ and its modulus can be got from (15).

$$
\left\{\begin{array}{l}
\bar{X}=(\bar{A})^{-1} \bar{Y} \\
\|\bar{X}\|=\left[(\Delta x)^{2}+(\Delta y)^{2}+(\Delta z)^{2}\right]^{1 / 2}
\end{array}\right.
$$

Once the modulus is obtained, it will be used to compare with a threshold to judge whether the process of positioning calculation is achieved or not. If it is larger than the threshold, the approximate coordinate should be modified according to the underlying expressions.

$$
\overline{\boldsymbol{\zeta}}^{1}=\overline{\boldsymbol{\zeta}}+\Delta \boldsymbol{\varsigma}, \quad \varsigma=x, y, z
$$

After obtaining the modified position, we consider it as the new approximate position and make use of it to perform the function of positioning calculation through iteration. Until the modulus of the estimated vector is lower than the given threshold, the uninterrupted positioning results can be fixed from three satellites.

Based on the above mentioned procedures, Fig.2 depicts the flowchart of the GPS positioning using the clock-aided method.

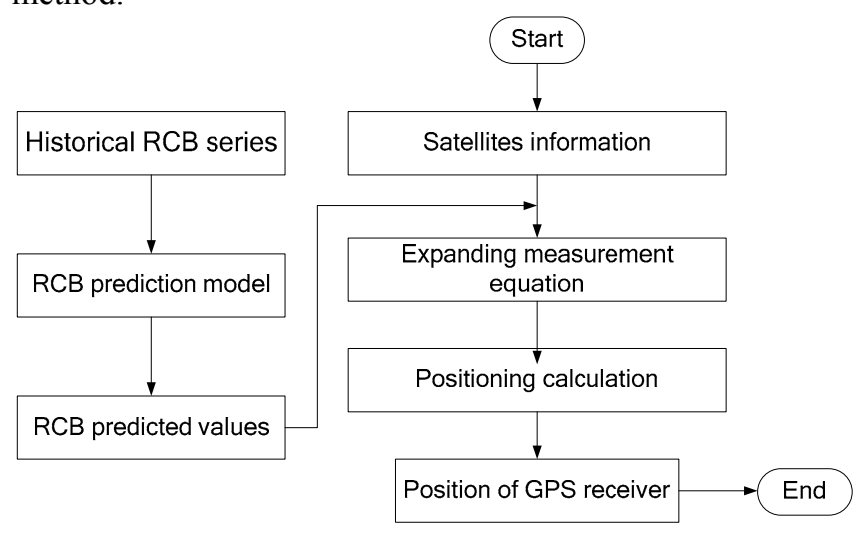

Fig.2 Flowchart of GPS positioning using clock-aided method.

\section{EXPERIMENTAL RESULTS AND DISCUSSIONS}

In this section, we investigate the positioning performance of the presented method in predicting RCB series and fixing the position of GPS receiver by some experiments. In these experiments, the static and dynamic tests with GPS receiver are discussed respectively. The original data were obtained from an OEM board with sampling frequency $1 \mathrm{~Hz}$, and there were four visible satellites. Furthermore, the positioning results brought out by the normal positioning method are considered as the correct ones.

In an urban environment with many tall buildings around, the situations wherein only three visible satellites only last for a few seconds. Accordingly, the method introduced in the paper can predict the subsequent values of RCB series by 
using the historical data firstly, and then the predicted values are introduced into GPS receiver to perform the function of positioning calculation with three visible satellites.

\section{A. Static Experiment Performance of the Method}

This section analyzes the performance of the presented method under static experiment. In this part, GPS receiver kept stationary, and it lasted for 120s. Firstly, the four estimated parameters of GPS receiver were calculated by the normal positioning method. Secondly, the numbers of visible satellites decreased from four to three between $91 \mathrm{~s}$ and $120 \mathrm{~s}$. In this case, the 90 historical data in the RCB series were adopted to build the prediction model, and then the 30 subsequent predicted values were obtained through the model. After obtaining the predicted values, the positioning information by using the clock-aided method is given.

\section{1) Prediction Results of $R C B$ series}

By using the combined prediction model, Fig.3 illustrates the predicted errors of the RCB series. It is observed that the maximal value among the predicted errors is less than 8 meters. In addition, the predicted errors cannot increase with the increment of prediction interval on the whole. That is to say, the combined prediction model in the paper is suitable for predicting the RCB series.

In fact, the combined prediction model presented in the paper decomposes the RCB series into several components including a LFC and several HFCs firstly. Based on the particular characteristic of different components, the PM and AR models are established in them respectively. Finally, the predicted values from the above single models are integrated together for obtaining the final predicted values of the RCB series. The combined prediction model can capture the characteristics and advantages of these single models, and thus may improve the prediction precision.

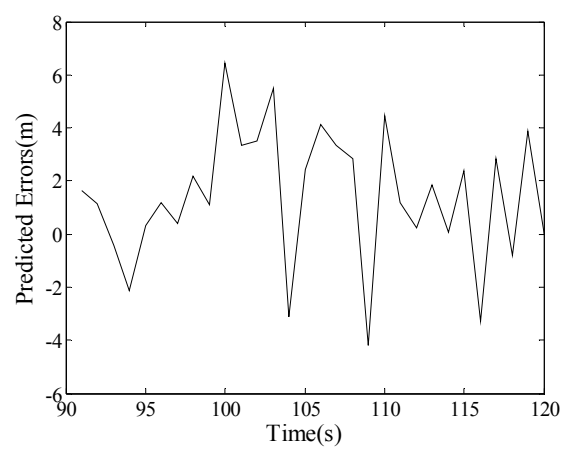

Fig.3 The predicted errors of the RCB series.

\section{2) Positioning Performance of the Clock-aided Method}

After the predicted values of the RCB series are obtained, they are used for calculating the position of GPS receiver through the clock-aided method in the paper. The threedimensional positioning errors (ErrX, ErrY and ErrZ) of GPS receiver are illustrated in Fig.4. It is seen from Fig.4 that the positioning errors in three dimensions are less than 10 meters. That is to say, the clock-aided positioning method can not only improve the continuity and reliability of GPS positioning, but also can implement the function of threedimensional positioning calculation under the conditions of only three visible satellites.
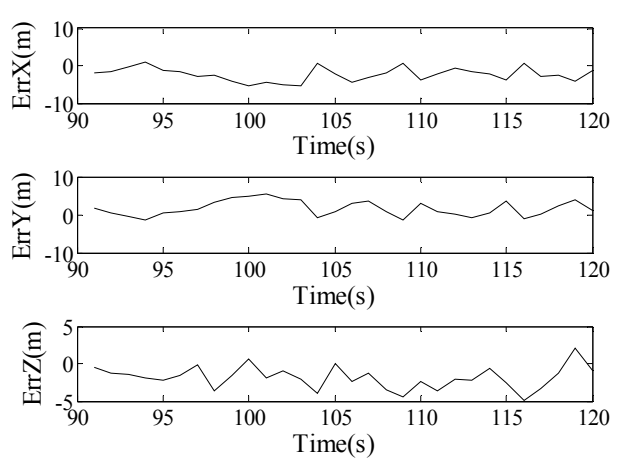

Fig.4 The three-dimensional positioning errors.

When the condition of the normal positioning method can not be satisfied, the clock-aided positioning methodology presented in the paper can obtain the positioning results by considering the predicted RCB values as one visible satellite. Consequently, the positioning performance of GPS receiver is related with the prediction precision of the RCB series. In order to analyze the relations between them, Fig.5 illustrates the absolute predicted errors of the RCB series together with the positioning errors of GPS receiver. It is shown that the more accurate the predicted values of the RCB series, the smaller the positioning errors of GPS receiver.
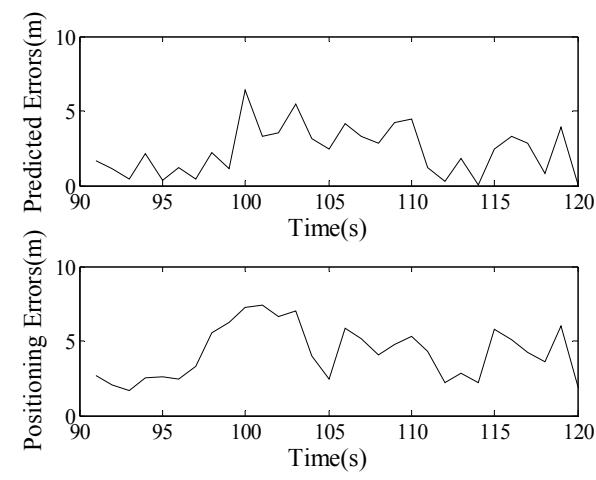

Fig.5 The predicted errors and positioning errors.

The reason of this phenomenon is that the clock-aided positioning method takes advantage of the predicted RCB value to calculate the expended measurement vector, and then the expended measurement vector is utilized for the positioning calculation. In the above process, the prediction precision of the RCB value plays an important part in the precision of the expended measurement vector, and then influences the positioning performance of GPS receiver as a result. When the prediction error of the predicted $\mathrm{RCB}$ value is smaller, the precision of the expended measurement vector is higher. In the circumstances, the positioning results of GPS receiver using the clock-aided method provided by these values are more accurate.

\section{B. Dynamic Experiment Performance of the Method}

A dynamic experiment is investigated to demonstrate the capability of the presented method. In the experiment, the GPS receiver moved along with a straight line and its speed was $5 \mathrm{~m} / \mathrm{s}$. In order to assess the performance of the presented method under the conditions of the dynamic experiment, this section makes use of the 90 historical data of the RCB series to obtain the 30 subsequent predicted values in the RCB series. the predicted errors of the RCB series and the 
positioning errors of GPS receiver are shown in Fig.6. From this figure, we can see that:

(1) When the length of the situations with only three visible satellites is shorter than $30 \mathrm{~s}$, the predicted errors are less than 12 meters. Consequently, the whole positioning errors of GPS receiver are less than 18 meters and in the range of normal positioning precision.

(2) Similar to the static experiment, the positioning precision accords with the prediction precision of the RCB series. The lower predicted errors of the RCB series mean that there are better positioning results of GPS receiver. Otherwise, the positioning precision becomes worse. The reason of this phenomenon is that the predicted error in the $\mathrm{RCB}$ series is the dominant error which will decrease the positioning precision after other errors have been properly modeled and compensated.

(3) Compared with the static status, the prediction precision of the RCB series and the positioning precision of GPS receiver under the conditions of the dynamic status are lower than the corresponding ones.
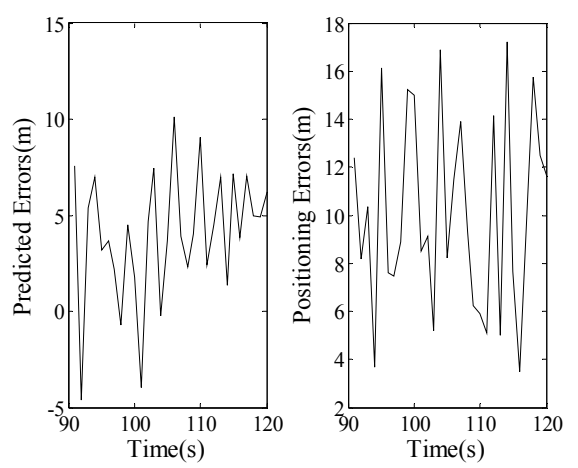

Fig.6 Forecasting errors of receiver clock bias.

From the experiments in this section, we can come to a conclusion that the combined prediction model in the paper is suitable for predicting the RCB series, and the clock-aided positioning method can provide uninterrupted and reliable positioning results which satisfy the precision requirement of GPS positioning in an urban environment. Additionally, the presented methodology does not need extra equipments, and it's much more feasible and effective. Equipped with the clock-aided positioning method in the paper, the positioning continuity and reliability of GPS receiver can be ensured for a few seconds even if there are only three satellites. The research work in the paper paves the way for positioning calculation, and should be valuable in the project application for improving the positioning continuity and reliability of GPS receiver in an urban environment.

\section{CONCLUSION}

In the paper, the three-dimensional positioning problem of GPS receiver in an urban environment has been discussed. Based on the characters of the RCB series, the combined prediction model on the basis of the theory of time series analysis is established and the clock-aided positioning method is presented to implement the function of positioning calculation. Experimental results demonstrate the method presented in the paper can provide a reliable and continuous positioning result without extra equipments under the conditions of only three visible satellites. Furthermore, the prediction precision of the RCB series has important effect on the positioning performance of GPS receiver. How to improve the prediction model and how to evaluate the relations between the positioning precision and prediction precision quantitatively are two important issues worthy of further research.

\section{REFERENCES}

[1] V. P. Alexandre, S. Nel, "Interference mitigation in a repeater and pseudolite indoor positioning system," IEEE Journal of Selected Topics in Signal Processing, vol. 3, no.5, October 2009, pp. 810-820.

[2] T. H. Chang, L. S. Wang, F. R. Chang, "A solution to ill-conditioned GPS positioning problem in an urban environment," IEEE

Transactions on Intelligent Transportation, vol. 10, no.1, March 2009, pp. $135-145$.

[3] R. Zheng, J. Chen, "New algorithm of GPS positioning in incomplete condition of temporal insufficient signal," Journal of University of Electronic Science and Technology, vol. 38, no.4, July 2009, pp. 496-500. (in Chinese).

[4] S. S. Jan, D. G. Egziabher, T. Walter, "Improving GPS-Based landing system performance using an empirical barometric altimeter confidence bound," IEEE Transactions on Aerospace and Electronic System, vol. 44, no.1, January 2008, pp. 127-146.

[5] S. Hong, M. H. Lee, S. H. Kwon, and H. H. Chun, "A car test for the estimation of GPS/INS alignment errors," IEEE Transactions on Intelligent Transportation System, vol. 5, no.3, September 2004, pp. 208-218.

[6] C. Liu, J. X. Gao, J. Wang, and Y. Liu, "GPS/Pseudolites technology for the slope deformation monitoring in open-pit mine," Journal of China Coal Society, vol. 35, no.5, May 2010, pp. 755-759.

[7] E. J. Sun, A. Nieto, Z. X. Li, "GPS and Google earth based 3D assisted driving system for trucks in surface mines," Mining Science and Technology, vol. 20, no.1, January 2010, pp. 138-142.

[8] S. Bednarz, P. Misra, "Receiver clock-based integrity monitoring for GPS precision approaches," IEEE Transactions on Aerospace and Electronic Systems, vol. 42, no.2, April 2006, pp. 636-643.

[9] H. L. Sun, Y. H. Jin, Y. D. Cui, and S. D. Cheng, "Network traffic prediction by a wavelet-based combined model," Chinese Physics B, vol. 18 , no.11, November 2009, pp. 4760-4768.

[10] S. G. Mallat, "A theory for multiresolution signal decomposition: the wavelet representation," IEEE Transactions on Pattern Analysis and Machine Intelligence, vol. 11, no.7, July 1989, pp. 674-693.

[11] A. Mathew, et al, "Detection and classification of voltage swells using wavelet transforms," International Journal of Computer and Electronic Engineering, vol. 2, no.3, June 2010, pp. 394-398.

[12] B. L. Zhang, R. Coggins, "Multiresolution forecasting for future trading using wavelet decomposition. Neural Networks," IEEE Transactions on Neural Networks, vol. 12, no.4, July 2001, pp. $765-775$.

Yunlong Teng received the M.S. degree in Operational research and cybernetics from University of Electronic Science and Technology of China in 2008. He is currently working towards the Ph.D. degree in Measuring and Testing Technology \& Instruments at the same university. His research interests are focused on test theory and technology, GPS navigation and positioning.

Yibing Shi received the B.S. degree in Radio Engineering, M.S. degree in Circuit and System, and Ph.D. degree in Measuring and Testing Technology \& Instruments, all from the University of Electronic Science and Technology of China, in 1985, 1988, and 2002, respectively. Now he is working as a professor and $\mathrm{Ph} . \mathrm{D}$. supervisor at the same university. His research interests include computer-aided test of VLSI and electronic systems, the theory and design of electronic measurement instruments, the research on well logging and the internet-based test.

Zhi Zheng received the M.S. degree in Circuit and Systems from University of Electronic Science and Technology of China in 2007. Currently, he is pursuing the Ph.D. degree in Communication and Information systems at the same university. His research interests are focused on array signal processing, smart antenna and wireless communication. 